\title{
Hausdorff Measures and Hausdorff Dimensions of the Invariant Sets for Iterated Function Systems of Geometric Fractals
}

\author{
Md. Jahurul Islam ${ }^{1, *}$, Md. Shahidul Islam ${ }^{1}$, Md. Shafiqul Islam² \\ ${ }^{1}$ Department of Mathematics, University of Dhaka, Bangladesh \\ ${ }^{2}$ School of Mathematics and Computational Science, University of Prince Edward Island, Canada
}

Copyright $\odot 2018$ by authors, all rights reserved. Authors agree that this article remains permanently open access under the terms of the Creative Commons Attribution License 4.0 International License

\begin{abstract}
In this paper, we discuss Hausdorff measure and Hausdorff dimension. We also discuss iterated function systems (IFS) of the generalized Cantor sets and higher dimensional fractals such as the square fractal, the Menger sponge and the Sierpinski tetrahedron and show the Hausdorff measures and Hausdorff dimensions of the invariant sets for IFS of these fractals.
\end{abstract}

Keywords Hausdorff Measure, Hausdorff Dimension, Invariant Set, Iterated Function System

\section{Introduction}

Any fractal has some infinitely repeating pattern. When crating such fractal, repetition of a certain series of steps is necessary which create that pattern. Iterated Function System is another way of generating fractals. It is based on taking a point or a figure and substituting it with several other identical ones. Iterated function system represents an extremely versatile method for conveniently generating a wide variety of useful fractal structures [1].

In this paper, we study the Cantor set and formulate iterated function system with probabilities of the generalized Cantor sets and also show their invariant measures using Markov operator and Barnsley-Hutchison multifunction [2]. We formulate iterated

function system of two dimensional the square fractal and the three dimensional fractals such as the Menger sponge, the Sierpinski tetrahedron. We also discuss Hausdorff measures and Hausdorff dimensions of the invariant sets for iterated function systems of these fractals.

The Iterated Function System is based on the application of a series of Affine Transformations. An Affine Transformation is a recursive transformation of the type [3]

$$
\left(\begin{array}{l}
x_{n+1} \\
y_{n+1}
\end{array}\right)=\left(\begin{array}{ll}
a & b \\
c & d
\end{array}\right)\left(\begin{array}{l}
x_{n} \\
y_{n}
\end{array}\right)+\left(\begin{array}{l}
e \\
f
\end{array}\right)
$$

where $a, b, c$ and $d$ control rotation and scaling, while $e$ and $f$ control linear translation.

Now we consider $w_{1}, w_{2}, \ldots w_{N}$ as a set of affine linear transformations, and let $A$ be the initial geometry.

Then a new geometry can be represented by

$$
F(A)=\bigcup_{i=1}^{N} w_{i}(A)
$$

where $F$ is known as the Barnsley-Hutchinson operator $[3,4]$.

We can also define iterated function system as follows:

Let $0<\beta<1$. Let $p_{1}, p_{2}, \ldots, p_{N}$ be points in the plane. Let $A_{i}(p)=\beta\left(p-p_{i}\right)+p_{i}$, where $p=\left(\begin{array}{l}x \\ y\end{array}\right)$ and $i=1,2,3, \ldots, N$. The collection of functions $\left\{A_{1}, A_{2}, \ldots, A_{N}\right\}$ is called an iterated function system [5].

In Section 2, we present a brief review on Hausdorf measure, their properties and Hausdorff dimension. Moreover, we present Hausdorff dimension of the invariant set for contracting maps. In Section 3, we present Hausdorff measures and Hausdorff dimensions of the generalized cantor sets. Hausdorff measures and Hausdorff dimensions of the invariant sets for IFS of the generalized cantor sets are presented in Section 4. In Section 5, we present some examples of two dimensional fractals and determine Hausdorff measures and Hausdorff dimensions of the invariant sets for IFS of these fractals. In Section 6 , we present examples of Hausdorff measures and Hausdorff dimensions of the invariant sets for IFS of three dimensional fractals. 


\section{Preliminaries}

\section{1. [6] Hausdorff Measure}

Let $U$ be any non-empty subset of the $n$-dimensional Euclidean space $\mathbf{R}^{n}$. The diameter of $U$ is defined as $|U|:=\sup \{|x-y|: x, y \in U\}$.

Here we will use the Euclidean metric:

$$
|x-y|:=\left(\left(x_{1}-y_{1}\right)^{2}+\left(x_{2}-y_{2}\right)^{2}+\cdots+\left(x_{n}-y_{n}\right)^{2}\right)^{1 / 2} \text {. }
$$

However, as will be shown shortly, we may use any $L_{p}$ metric. If $E \subseteq \mathbf{R}^{n}$, and a collection $\left\{U_{i}\right\}_{i \in I}$ satisfies the following conditions:

1. $\left|U_{i}\right| \leq \delta$ for each $i \in I$;

2. $E \subseteq \bigcup_{i \in I} U_{i}$,

then we say the collection is a $\delta$-cover of $E$. We may assume the collection is always countable.

Definition 2.2. [7] Let $E \in \mathbf{R}^{n}$ be a Borel set with $\left\{U_{i}\right\}_{i \in I}$ a $\delta$-cover for it. Given any $s>0$, we define the $\delta$-approximating s-dimensional Hausdorff measure $\mathcal{H}_{\delta}^{s}: \mathbf{R}^{n} \rightarrow \mathbf{R}$ as follows

$$
\begin{aligned}
& { }_{\mathcal{H}}^{s}(E)=\inf \left\{\sum_{i=1}^{\infty}\left|U_{i}\right|^{s}:\left\{U_{i}\right\}_{i \in I}\right. \text { forms a } \\
& \delta \text {-cover for } E\} .
\end{aligned}
$$

If $\delta \rightarrow 0$, then we have a more succinct formula $\mathcal{H}^{s}(E)=\lim _{\delta \rightarrow 0}\left(\mathcal{H}_{\delta}^{s}(E)\right)$

gives the s-dimensional Hausdorff measure of $E$.

Definition 2.3. A mapping $S: \mathbf{R}^{n} \rightarrow \mathbf{R}^{n}$ is a similarity with ratio $r>0$ if

$$
|S(x)-S(y)|=r|x-y| .
$$

Definition 2.4. Let $(M, d)$ be a metric space. A mapping $f: M \rightarrow M$ is a contraction or contractor with the property that there is some nonnegative real number such that for all $x$ and $y$ in $M$,

$$
d(f(x), f(y)) \leq k d(x, y) .
$$

The smallest such value of $k$ is called the Lipschitz constant of $f$. Contractive maps are sometimes called Lipschitzian maps.

\subsection{Properties of Hausdorff Measure}

Theorem 2.5.1. (Outer Measure) The following are true for any metric space (i) (Null Empty set) $\mathcal{H}^{s}(\phi)=0$.

(ii) (Monotonicity) If $E \subseteq F$, then

$$
\mathcal{H}^{s}(E) \leq \mathcal{H}^{s}(F)
$$

(iii) (Countable Subadditivity)

$$
{ }_{\mathcal{H}}^{s}\left(\bigcup_{i=1}^{\infty} E_{i}\right) \leq \sum_{i=1}^{\infty} \mathcal{H}^{s}\left(E_{i}\right) .
$$

Proof: The proof can be found in [8].

Theorem 2.5.2. (Countable Additivity)

Let $\left\{E_{i}\right\}_{i=1}^{\infty}$ be a countable collection of disjoint $\mathcal{H}^{s}-$ measurable subsets of $X$. Then we have

$$
{ }_{\mathcal{H}}^{s}\left(\bigcup_{i=1}^{\infty} E_{i}\right)=\sum_{i=1}^{\infty} \mathcal{H}^{s}\left(E_{i}\right) .
$$

Proof: The proof can be found in [9].

Theorem 2.5.3. [7] (Scaling Property).

Let $S: \mathbf{R}^{n} \rightarrow \mathbf{R}^{n}$ be a similarity mapping, and any $\lambda>0$ such that $|S(x)-S(y)| \leq \lambda|x-y|$ for any $x, y \in \mathbf{R}^{n}$, where $\lambda$ is the scaling factor. If $E \in \mathbf{R}^{n}$, then $\mathcal{H}^{s}\left(S(E)=\lambda^{s}{ }_{\mathcal{H}}^{s}(E)\right.$.

Proof: Let $\left\{U_{i}\right\}_{i=1}^{\infty}$ be a $\delta$ - cover of $E$. Then $\left\{S\left(U_{i}\right)\right\}_{i=1}^{\infty}$ is a $\lambda \delta$-cover of $S(E)$. Thus we see that, given $s>0$ :

$$
\mathcal{H}_{\lambda \delta}^{s}(S(E))=\inf \left\{\sum_{i=1}^{\infty}\left|S\left(U_{i}\right)\right|^{s}: \quad\left\{U_{i}\right\}_{i=1}^{\infty}\right. \text { forms a }
$$

$\lambda \delta$-cover $\}$

Here $\sum_{i=1}^{\infty}\left|S\left(U_{i}\right)\right|^{s} \leq \sum_{i=1}^{\infty}\left|\lambda U_{i}\right|^{s}=\lambda^{s} \sum_{i=1}^{\infty}\left|U_{i}\right|^{s}$

We obtain

$$
\mathcal{H}_{\lambda \delta}^{s}(S(E)) \leq \lambda^{s} \mathcal{H}_{\delta}^{s}(E)
$$

So, letting $\delta \rightarrow 0$ gives that

$$
\mathcal{H}^{s}(S(E)) \leq \lambda^{s} \mathcal{H}^{s}(E) .
$$

Replacing $\lambda$ by $\frac{1}{\lambda}$ and $E$ by $S(E)$ gives the opposite inequality, as required.

Theorem 2.5.3. [7] (Scaling Property).

Let $S: \mathbf{R}^{n} \rightarrow \mathbf{R}^{n}$ be a similarity mapping, and any 
$\lambda>0$ such that $|S(x)-S(y)| \leq \lambda|x-y|$ for any $x, y \in \mathbf{R}^{n}$, where $\lambda$ is the scaling factor. If $E \in \mathbf{R}^{n}$, then $\mathcal{H}^{s}\left(S(E)=\lambda^{s} \mathcal{H}^{s}(E)\right.$.

Proof: Let $\left\{U_{i}\right\}_{i=1}^{\infty}$ be a $\delta$ - cover of $E$. Then $\left\{S\left(U_{i}\right)\right\}_{i=1}^{\infty}$ is a $\lambda \delta$-cover of $S(E)$. Thus we see that, given $s>0$ :

$\mathcal{H}_{\lambda \delta}^{s}(S(E))=\inf \left\{\sum_{i=1}^{\infty}\left|S\left(U_{i}\right)\right|^{s}:\left\{U_{i}\right\}_{i=1}^{\infty}\right.$ forms a $\lambda \delta$-cover $\}$

Here $\sum_{i=1}^{\infty}\left|S\left(U_{i}\right)\right|^{s} \leq \sum_{i=1}^{\infty}\left|\lambda U_{i}\right|^{s}=\lambda^{s} \sum_{i=1}^{\infty}\left|U_{i}\right|^{s}$

We obtain

$$
\mathcal{H}_{\lambda \delta}^{s}(S(E)) \leq \lambda^{s} \mathcal{H}_{\delta}^{s}(E) .
$$

So, letting $\delta \rightarrow 0$ gives that

$$
\mathcal{H}^{s}(S(E)) \leq \lambda^{s} \mathcal{H}^{s}(E) .
$$

Replacing $\lambda$ by $\frac{1}{\lambda}$ and $E$ by $S(E)$ gives the opposite inequality, as required.

\subsection{4. [7] Hausdorff Dimension}

Let $E$ be a given set. Note that $\left.\mathcal{H}_{\delta}^{s}(E)\right)$ decreases as $s$ increases. This means that $\mathcal{H}^{s}(E)$ also decreases with $s$ increasing. If $t>s$ and $\left\{U_{i}\right\}$ is a $\delta$-cover of $E$, then each $\left|U_{i}\right|^{t-s} \leq \delta^{t-s}$ since $\left|U_{i}\right| \leq \delta$, so

$$
\begin{aligned}
& \sum_{i=1}^{\infty}\left|U_{i}\right|^{t}=\sum_{i=1}^{\infty}\left(\left|U_{i}\right|^{t-s}\left|U_{i}\right|^{s}\right) \\
& \leq \sum_{i=1}^{\infty}\left(\delta^{t-s}\left|U_{i}\right|^{s}\right) \leq \delta^{t-s} \sum_{i=1}^{\infty}\left|U_{i}\right|^{s} .
\end{aligned}
$$

After taking the infima over all $\delta$-covers, we can easily see that

$$
\mathcal{H}_{\delta}^{t}(E) \leq \delta^{t-s} \mathcal{H}_{\delta}^{s}(E)
$$

Let $\delta \rightarrow 0$. Then $\mathcal{H}_{\delta}^{t}(E) \rightarrow 0$ if $\mathcal{H}^{s}(E)$ is finite. Also if $\mathcal{H}_{\delta}^{t}$ is bounded and finite then

$$
\mathcal{H}_{\delta}^{s}(E) \rightarrow \infty .
$$

Two applications of equation (1) should be noted:
1. If $\mathcal{H}^{s}(E)<\infty$ and $t>s$, then $\mathcal{H}^{t}(E)=0$.

Proof: Equation (1) shows that

$\mathcal{H}_{\delta}^{t}(E) \leq \delta^{t-s} \mathcal{H}_{\delta}^{s}(E)$ for any positive $\delta$.

The result follows after taking limits, since

$$
\mathcal{H}^{s}(E)<\infty \text {. }
$$

2. If $\mathcal{H}^{s}(E)>0$ and $t<s$, then $\mathcal{H}^{t}(E)=\infty$.

Proof: Equation (1) shows that

$\frac{1}{\delta^{t-s}} \mathcal{H}_{\delta}^{t}(E) \leq \mathcal{H}_{\delta}^{s}(E)$ for any positive $\delta$. After taking limits, we see that $\mathcal{H}^{t}(E)=\infty$, since $\lim _{\delta \rightarrow 0} \frac{1}{\delta^{t-s}}=\infty$ and

$$
\lim _{\delta \rightarrow 0} \mathcal{H}_{\delta}^{s}(E)=\mathcal{H}^{s}(E)>0 .
$$

One immediate consequence of these observation is that

$$
s(E)= \begin{cases}\infty & \text { if } s<\operatorname{dim}_{H}(E) \\ 0 & \text { if } s>\operatorname{dim}_{H}(E) \\ \text { finite, nonzero if } s=\operatorname{dim}_{H}(E)\end{cases}
$$

\section{Hausdorff Dimension of the Invariant set for \\ Contraction Maps}

Let $S_{1}, S_{2}, \ldots, S_{N}$ be contractions. A subset $F$ of $X$ is called invariant for the transformation $S_{i}$ if

$$
F=\bigcup_{i=1}^{N} S_{i}(F)
$$

In the case where $S_{i}: X \rightarrow X$ are similarities with Lipschitz constants $L_{i}$ for $i=1,2, \ldots, N$ respectively, a theorem proved by M. Hata (Theorem 10.3 of [9] and Proposition 9.7 of [6]) allows us to calculate the Hausdorff dimension of the invariant set for $S_{1}, S_{2}, \ldots, S_{N}$. Namely, if we assume that $F$ is an invariant set for the similarities $S_{1}, S_{2}, \ldots, S_{N}$ and $S_{i}(F) \cap S_{j}(F)=\phi$ for $i \neq j$, then

$\operatorname{dim}_{H} F=s$, where $s$ is given by

$$
\sum_{i=1}^{N} L_{i}^{s}=1 .
$$




\section{Dimensions of the Generalized Cantor Sets}

Let $C_{1 / 3}$ be the Cantor middle $\frac{1}{3}$ set. The set $C_{1 / 3}$ splits into a left part $\left(C_{1 / 3}\right)_{L}=C_{1 / 3} \cap\left[0, \frac{1}{3}\right]$ and a right part $\left(C_{1 / 3}\right)_{R}=C_{1 / 3} \cap\left[\frac{2}{3}, 1\right]$.

Clearly, both parts are geometrically similar to $C_{1 / 3}$ but scaled by a ratio $\frac{1}{3}$ and $C_{1 / 3}=\left(C_{1 / 3}\right)_{L} \cup\left(C_{1 / 3}\right)_{R}$ with this union disjoint.

Thus by the scaling property we have

$$
\begin{aligned}
& \mathcal{H}^{s}\left(C_{1 / 3}\right)=\mathcal{H}^{s}\left(\left(C_{1 / 3}\right)_{L}\right)+\mathcal{H}^{s}\left(\left(C_{1 / 3}\right)_{R}\right) \\
& =\left(\frac{1}{3}\right)^{s} \mathcal{H}^{s}\left(C_{1 / 3}\right)+\left(\frac{1}{3}\right)^{s} \mathcal{H}^{s}\left(C_{1 / 3}\right)
\end{aligned}
$$$$
\text { (assume } 0<\mathcal{H}^{s}\left(C_{1 / 3}\right)<\infty \text { when } \mathrm{s}=\operatorname{dim}_{H} F \text { ) }
$$

Now cancelling $\mathcal{H}^{s}\left(C_{1 / 3}\right)$ form both sides, we have

$$
\begin{aligned}
1 & =\left(\frac{1}{3}\right)^{s} \quad+\left(\frac{1}{3}\right)^{s} \quad \Rightarrow 1=2 \times 3^{-s} \\
\therefore s & =\frac{\log 2}{\log 3}=0.631
\end{aligned}
$$

Thus the Hausdorff dimension of the Cantor middle $\frac{1}{3}$ set is $\operatorname{dim}_{H} C_{1 / 3}=0.631$.

Since the Cantor middle $\frac{1}{3}$ set is in $\mathbf{R}^{1}$, $\operatorname{dim}_{H} C_{1 / 3}=0.631<1, \quad \mathcal{H}^{s}\left(C_{1 / 3}\right)=0$.

Hence the Hausdorff measure of the Cantor middle $\frac{1}{3}$ set is zero.

Similarly, we can show that the Hausdorff dimension of the Cantor middle $\frac{1}{5}$ set is

$\operatorname{dim}_{H} C_{1 / 5}=0.682$.

Since $\operatorname{dim}_{H} C_{1 / 5}=0.682<1, \mathcal{H}^{s}\left(C_{1 / 5}\right)=0$.

We obtain the Hausdorff measure of the Cantor middle $\frac{1}{5}$ set is zero. In general, we can show that the
Hausdorff dimension of the Cantor middle $\frac{1}{2 m-1}$ $(2 \leq m<\infty)$, set is

$\operatorname{dim}_{H} C_{1 /(2 m-1)}=\frac{\log m}{\log (2 m-1)}$.

Since $\operatorname{dim}_{H} C_{1 / 2 m-1)}=\frac{\log m}{\log (2 m-1)}<1$,

$\mathcal{H}^{s}\left(C_{1 /(2 m-1)}\right)=0$ for $2 \leq m<\infty$.

Hence the Hausdorff measure of the generalized Cantor sets is zero.

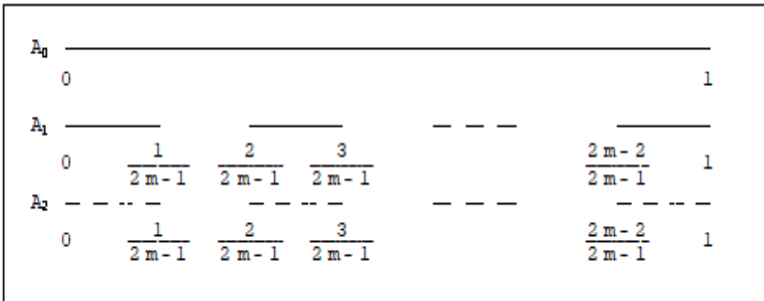

Figure 1. Construction of the Cantor middle $\frac{1}{2 m-1}$ set as IFS

\section{Hausdorff Measures and Hausdorff Dimensions of the Invariant Sets for IFS of the Generalized Cantor Sets}

Iterated Function System of the Generalized Cantor Sets: Let $X=[0,1]$. Let $(X, \rho)$ be a complete separable metric space. If $w_{k}: X \rightarrow X$ is a function which is defined by

$$
w_{k}(x)=\frac{x}{2 m-1}+\frac{2(k-1)}{2 m-1}, m \geq 2
$$

with contracting factor $L=\frac{1}{2 m-1},(2 \leq m<\infty)$ for $k=1,2, \ldots, m$ respectively, then the family $\left\{w_{k}: k=1,2, \ldots, m\right\}$ is called an iterated function system of the generalized Cantor sets (IFSGCS) which is denoted by the Cantor middle $\frac{1}{2 m-1},(2 \leq m<\infty)$ sets.

Let $F$ be an invariant set for IFS of the Cantor middle $\frac{1}{2 m-1}$ set. If $F \subseteq \mathbf{R}$, then $w_{i}(F) \cap w_{j}(F)=\phi$ for $i \neq j$, and $L_{i}=\frac{1}{2 m-1}$ for $i=1,2, \ldots, m$.

Now from (2), we have 


$$
\begin{aligned}
& \quad m \cdot\left(\frac{1}{2 m-1}\right)^{s}=1 \Rightarrow s \log (2 m-1)=\log m \\
& \therefore s=\frac{\log m}{\log (2 m-1)},(2 \leq m<\infty) .
\end{aligned}
$$

Thus the Hausdorff dimension of the invariant set for IFS of the generalized Cantor sets is

$$
\operatorname{dim}_{H} F=\frac{\log m}{\log (2 m-1)},(2 \leq m<\infty) .
$$

Hence the Hausdorff measures of the generalized Cantor sets is zero.

\section{Hausdorff Measures and Hausdorff \\ Dimensions of the Invariant Sets for IFS of Two Dimensional Fractals}

\subsection{Hausdorff Measure and Dimension of the Invariant Set for IFS of the Box Fractal}

Let $B$ be an invariant set for IFS of the box fractal which is defined by the following

$$
\begin{aligned}
& w_{1}(x, y)=\left(\frac{1}{3} x, \frac{1}{3} y\right), w_{2}(x, y)=\left(\frac{1}{3} x+\frac{2}{3}, \frac{1}{3} y\right), \\
& w_{3}(x, y)=\left(\frac{1}{3} x, \frac{1}{3} y+\frac{2}{3}\right), \\
& w_{4}(x, y)=\left(\frac{1}{3} x+\frac{2}{3}, \frac{1}{3} y+\frac{2}{3}\right), \\
& w_{5}(x, y)=\left(\frac{1}{3} x+\frac{1}{3}, \frac{1}{3} y+\frac{1}{3}\right)
\end{aligned}
$$

Now we have

$$
\begin{aligned}
& \rho\left(w_{1}(x, y), w_{1}\left(x^{\prime}, y^{\prime}\right)\right) \\
= & \sqrt{\frac{1}{9}\left(x-x^{\prime}\right)^{2}+\frac{1}{9}\left(y-y^{\prime}\right)^{2}} \\
= & \frac{1}{3} \sqrt{\left(x-x^{\prime}\right)^{2}+\left(y-y^{\prime}\right)^{2}} \\
= & \frac{1}{3} \rho\left((x, y),\left(x^{\prime}, y^{\prime}\right)\right)
\end{aligned}
$$$$
\text { It follows that } w_{1}, w_{2}, w_{3}, w_{4} \text { and } w_{5} \text { are }
$$
contraction on $\mathbf{R}^{2}$ with $L=\frac{1}{3}$.

$$
\text { If } B \subseteq \mathbf{R}^{2} \text {, then } \bigcap_{i=1}^{5} w_{i}(B)=\phi \text { and } L_{i}=\frac{1}{3} \text { for }
$$
each $i=1,2,3,4,5$. Now from (2), we have

$$
\left(\frac{1}{3}\right)^{s}+\left(\frac{1}{3}\right)^{s}+\left(\frac{1}{3}\right)^{s}+\left(\frac{1}{3}\right)^{s}+\left(\frac{1}{3}\right)^{s}=1
$$

$$
\begin{aligned}
& \Rightarrow 3^{s}=5 \Rightarrow s \log 3=\log 5 \\
& \therefore s=\frac{\log 5}{\log 3}=1.46 .
\end{aligned}
$$

Thus the Hausdorff dimension of the invariant set for IFS of the box fractal is $\operatorname{dim}_{H} B=1.46$.

Since the box fractal is in $\mathbf{R}^{2}$, and $\operatorname{dim}_{H} B=1.46<2, \mathcal{H}^{s}(B)=0$.

Hence the Hausdorff measure of the invariant set for IFS of the box fractal is zero.

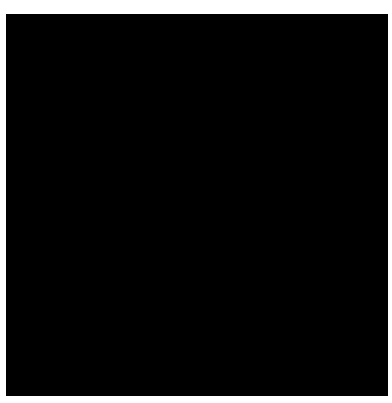

$B_{0}$

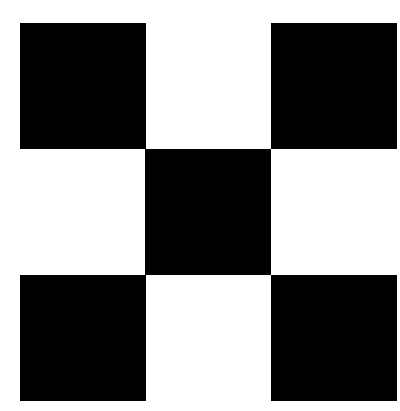

$$
B_{1}\left(1^{\text {st }} \text { Iteration }\right)
$$

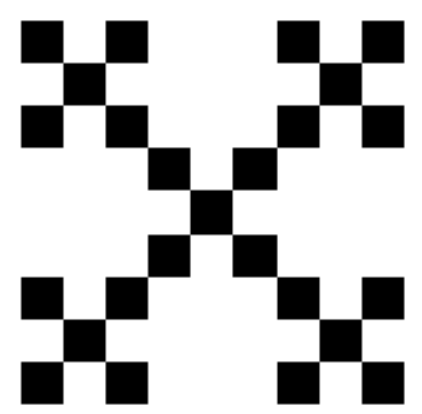

$$
B_{2\left(2^{\text {nd }} \text { Iteration }\right)}
$$

Figure 2. onstruction of the box fractal as IFS

5.2. Hausdorff Measure and Dimension of the Invariant Set for IFS of the Square Fractal (Using the Cantor 


\section{Middle $\frac{1}{3}$ Set)}

Let $S$ be an invariant set for IFS of the square fractal (using the Cantor middle $\frac{1}{3}$ set) which is represented by the following:

$$
\begin{aligned}
w_{1}(x, y) & =\left(\frac{1}{3} x, \frac{1}{3} y\right), w_{2}(x, y)=\left(\frac{1}{3} x+\frac{2}{3}, \frac{1}{3} y\right) \\
w_{3}(x, y) & =\left(\frac{1}{3} x, \frac{1}{3} y+\frac{2}{3}\right), \\
w_{4}(x, y)= & \left(\frac{1}{3} x+\frac{2}{3}, \frac{1}{3} y+\frac{2}{3}\right)
\end{aligned}
$$

We have

$$
\begin{aligned}
& \rho\left(w_{1}(x, y), w_{1}\left(x^{\prime}, y^{\prime}\right)\right) \\
= & \sqrt{\frac{1}{9}\left(x-x^{\prime}\right)^{2}+\frac{1}{9}\left(y-y^{\prime}\right)^{2}} \\
= & \frac{1}{3} \sqrt{\left(x-x^{\prime}\right)^{2}+\left(y-y^{\prime}\right)^{2}} \\
= & \frac{1}{3} \rho\left((x, y),\left(x^{\prime}, y^{\prime}\right)\right)
\end{aligned}
$$

It follows that $w_{1}, w_{2}, w_{3}$ and $w_{4}$ are contraction on $\mathbf{R}^{2}$ with $L=\frac{1}{3}$.

$$
\text { If } S \subseteq \mathbf{R}^{2} \text {, then } \bigcap_{i=1}^{4} w_{i}(S)=\phi \text { and } L_{i}=\frac{1}{3} \text { for }
$$

each $i=1,2,3,4$. Now from (2), we have

$$
\begin{aligned}
& \left(\frac{1}{3}\right)^{s}+\left(\frac{1}{3}\right)^{s}+\left(\frac{1}{3}\right)^{s}+\left(\frac{1}{3}\right)^{s}=1 \\
& \Rightarrow 3^{s}=4 \Rightarrow s \log 3=\log 4 \\
& \therefore s=\frac{\log 4}{\log 3}=1.26 .
\end{aligned}
$$

Thus the Hausdorff dimension of the invariant set for IFS of the square fractal is $\operatorname{dim}_{H} S=1.26$.
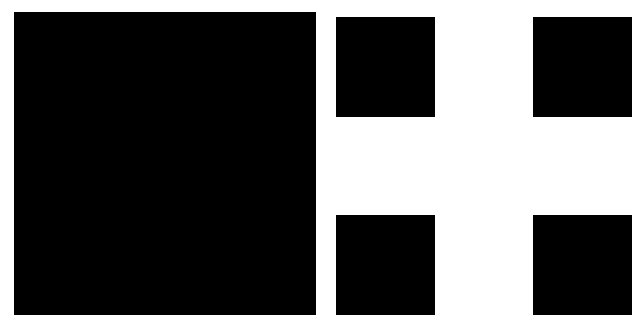

$$
S_{0} \quad S_{1}{\left(1{ }^{\text {st }} \text { Iteration }\right)}
$$

Figure 3. Construction of the square fractal (using the Cantor middle $\frac{1}{3}$ set) as IFS

Since the square fractal is in $\mathbf{R}^{2}$, and $\operatorname{dim}_{H} S=1.26<2$,

$$
\mathcal{H}^{s}(S)=0 \text {. }
$$

Hence the Hausdorff measure of the invariant set for IFS of the square fractal is zero.

\subsection{Hausdorff Measure and Dimension of the Invariant Set for IFS of the Square Fractal (Using the Cantor Middle $\frac{1}{5}$ Set)}

Let $F$ be an invariant set for IFS of the square fractal which is defined by the following:

$$
\begin{aligned}
w_{1}(x, y) & =\left(\frac{1}{5} x, \frac{1}{5} y\right), w_{2}(x, y)=\left(\frac{1}{5} x+\frac{2}{5}, \frac{1}{5} y\right), \\
w_{3}(x, y) & =\left(\frac{1}{5} x, \frac{1}{5} y+\frac{2}{5}\right), \\
w_{4}(x, y) & =\left(\frac{1}{5} x+\frac{4}{5}, \frac{1}{5} y\right), \\
w_{5}(x, y) & =\left(\frac{1}{5} x, \frac{1}{5} y+\frac{4}{5}\right), \\
w_{6}(x, y) & =\left(\frac{1}{5} x+\frac{2}{5}, \frac{1}{5} y+\frac{2}{5}\right),
\end{aligned}
$$$$
w_{7}(x, y)=\left(\frac{1}{5} x+\frac{2}{5}, \frac{1}{5} y+\frac{4}{5}\right),
$$$$
w_{8}(x, y)=\left(\frac{1}{5} x+\frac{4}{5}, \frac{1}{5} y+\frac{2}{5}\right),
$$$$
w_{9}(x, y)=\left(\frac{1}{5} x+\frac{4}{5}, \frac{1}{5} y+\frac{4}{5}\right)
$$

Now we obtain 


$$
\begin{aligned}
& \rho\left(w_{1}(x, y), w_{1}\left(x^{\prime}, y^{\prime}\right)\right) \\
& =\sqrt{\frac{1}{25}\left(x-x^{\prime}\right)^{2}+\frac{1}{25}\left(y-y^{\prime}\right)^{2}} \\
& =\frac{1}{5} \sqrt{\left(x-x^{\prime}\right)^{2}+\left(y-y^{\prime}\right)^{2}} \\
& =\frac{1}{5} \rho\left((x, y),\left(x^{\prime}, y^{\prime}\right)\right)
\end{aligned}
$$

It follows that $w_{1}, w_{2}, w_{3}, \ldots, w_{8}$ and $w_{9}$ are contraction on $\mathbf{R}^{2}$ with $L=\frac{1}{5}$.

If $S \subseteq \mathbf{R}^{2}$, then $\bigcap_{i=1}^{9} w_{i}(S)=\phi$ and $L_{i}=\frac{1}{5}$ for each $i=1,2,3, \ldots, 9$.

Now from (2), we have

$$
\begin{aligned}
& 9 \cdot\left(\frac{1}{5}\right)^{s}=1 \Rightarrow 5^{s}=9 \Rightarrow s \log 5=\log 9 \\
& \therefore s=\frac{\log 9}{\log 5}=1.36 .
\end{aligned}
$$

Thus the Hausdorff dimension of the invariant set for IFS of the square fractal is $\operatorname{dim}_{H} F=1.36$.

Since the square fractal is in $\mathbf{R}^{2}$, and $\operatorname{dim}_{H} S=1.36<2$,

$$
\mathcal{H}^{s}(S)=0 \text {. }
$$

Hence the Hausdorff measure of the invariant set for IFS of the square fractal is zero.
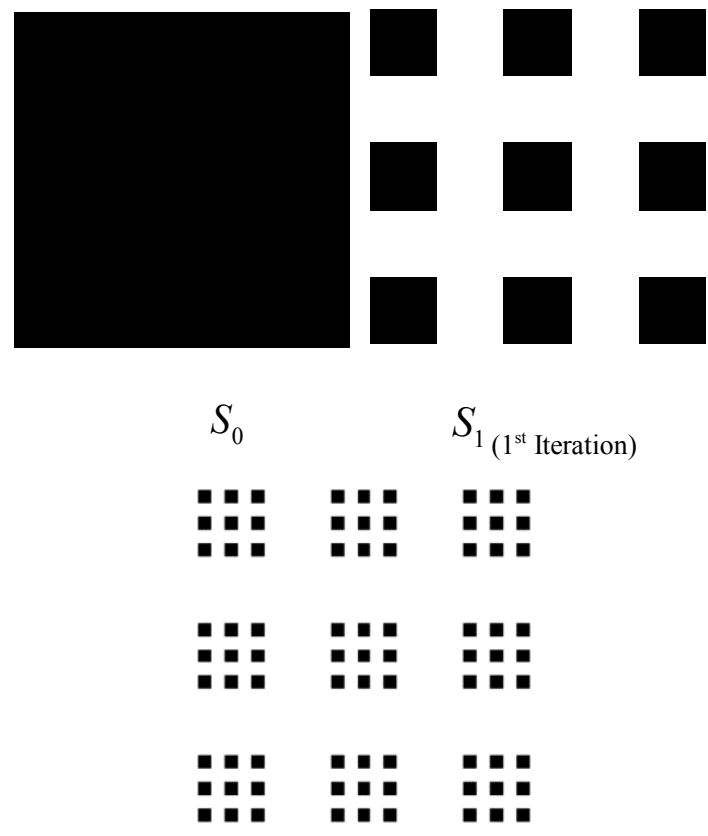

$$
S_{2\left(2^{\text {nd }} \text { Iteration }\right)}
$$

Figure 4. Construction of the square fractal (using the Cantor middle $\frac{1}{5}$
set) as IFS

\section{Hausdorff Measures and Hausdorff Dimensions of the Invariant Sets for IFS of Three Dimensional Fractals}

\subsection{Hausdorff Measure and Dimension of the Invariant Set for IFS of the Menger Sponge}

Let $M$ be an invariant set for IFS of the Menger sponge which is represented by the following:

$$
\begin{gathered}
w_{1}(x, y, z)=\left(\frac{1}{3} x, \frac{1}{3} y, \frac{1}{3} z\right), \\
w_{2}(x, y, z)=\left(\frac{1}{3}(x+1), \frac{1}{3} y, \frac{1}{3} z\right), \\
w_{3}(x, y, z)=\left(\frac{1}{3} x, \frac{1}{3}(y+1), \frac{1}{3} z\right), \\
w_{4}(x, y, z)=\left(\frac{1}{3}(x+1), \frac{1}{3} y, \frac{1}{3}(z+1)\right), \\
w_{5}(x, y, z)=\left(\frac{1}{3}(x+2), \frac{1}{3} y, \frac{1}{3} z\right), \\
w_{6}(x, y, z)=\left(\frac{1}{3} x, \frac{1}{3}(y+2), \frac{1}{3} z\right), \\
w_{7}(x, y, z)=\left(\frac{1}{3} x, \frac{1}{3} y, \frac{1}{3}(z+2)\right), \\
w_{8}(x, y, z)=\left(\frac{1}{3}(x+1), \frac{1}{3}(y+2), \frac{1}{3} z\right), \\
w_{12}(x, y, z)=\left(\frac{1}{3}(x+2), \frac{1}{3} y, \frac{1}{3}(z+1)\right), \\
w_{14}(x, y, z)=\left(\frac{1}{3}(x+1), \frac{1}{3} y, \frac{1}{3}(z+2)\right), \\
w_{10}(x, y, z)=\left(\frac{1}{3} x, \frac{1}{3}(y+1), \frac{1}{3}(z+2)\right), \\
w_{11}(x, y, z)=\left(\frac{1}{3}(x+2), \frac{1}{3}(y+1), \frac{1}{3} z\right), \\
\left.w_{1}(y+2), \frac{1}{3}(z+1)\right),
\end{gathered}
$$




$$
\begin{gathered}
w_{15}(x, y, z)=\left(\frac{1}{3}(x+2), \frac{1}{3} y, \frac{1}{3}(z+2)\right), \\
w_{16}(x, y, z)=\left(\frac{1}{3} x, \frac{1}{3}(y+2), \frac{1}{3}(z+2)\right), \\
w_{17}(x, y, z)=\left(\frac{1}{3}(x+2), \frac{1}{3}(y+2), \frac{1}{3}(z+1)\right), \\
w_{18}(x, y, z)=\left(\frac{1}{3}(x+2), \frac{1}{3}(y+1), \frac{1}{3}(z+2)\right), \\
w_{19}(x, y, z)=\left(\frac{1}{3}(x+1), \frac{1}{3}(y+2), \frac{1}{3}(z+2)\right), \\
w_{20}(x, y, z)=\left(\frac{1}{3}(x+2), \frac{1}{3}(y+2), \frac{1}{3}(z+2)\right) \text { Now }
\end{gathered}
$$

we have

$$
\begin{aligned}
& \rho\left(w_{1}(x, y, z), w_{1}\left(x^{\prime}, y^{\prime}, z^{\prime}\right)\right) \\
& =\sqrt{\frac{1}{9}\left(x-x^{\prime}\right)^{2}+\frac{1}{9}\left(y-y^{\prime}\right)^{2}+\frac{1}{9}\left(z-z^{\prime}\right)^{2}} \\
& =\frac{1}{3} \sqrt{\left(x-x^{\prime}\right)^{2}+\left(y-y^{\prime}\right)^{2}+\left(z-z^{\prime}\right)^{2}} \\
& =\frac{1}{3} p\left((x, y, x),\left(x^{\prime}, y^{\prime}, z^{\prime}\right)\right)
\end{aligned}
$$

It follows that $w_{1}, w_{2}, \ldots, w_{19}$ and $w_{20}$ are contraction on $\mathbf{R}^{3}$ with $L=\frac{1}{3}$.

If $M \subseteq \mathbf{R}^{3}$, then $\bigcap_{i=1}^{20} w_{i}(M)=\phi$ and $L_{i}=\frac{1}{3}$ for each $i=1,2, \ldots, 20$.

Now from (2), we have

$$
\begin{aligned}
& 20 \cdot\left(\frac{1}{3}\right)^{s}=1 \Rightarrow 3^{s}=20 \Rightarrow s \log 3=\log 20 \\
& \therefore s=\frac{\log 20}{\log 3}=2.726 .
\end{aligned}
$$

Thus the Hausdorff dimension of the invariant set for IFS of the Menger sponge is $\operatorname{dim}_{H} M=2.726$. Since the Menger sponge is in $\mathbf{R}^{3}, \quad$ and $\operatorname{dim}_{H} M=2.726<3$,

$$
\mathcal{H}^{s}(M)=0 \text {. }
$$

Hence the Hausdorff measure of the invariant set for IFS of the Menger sponge is zero.

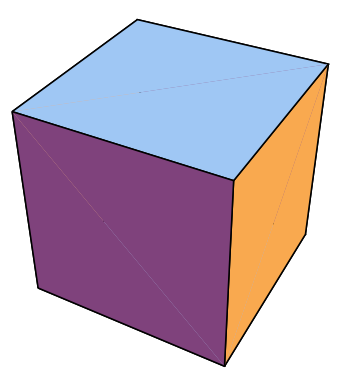

$M_{0}$

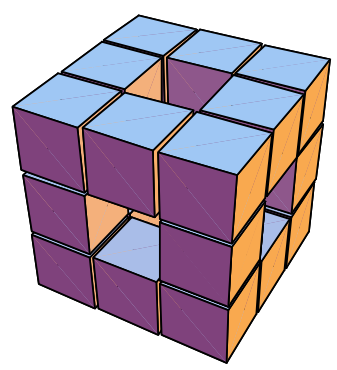

$M_{1\left(1^{\text {st }} \text { Iteration }\right)}$

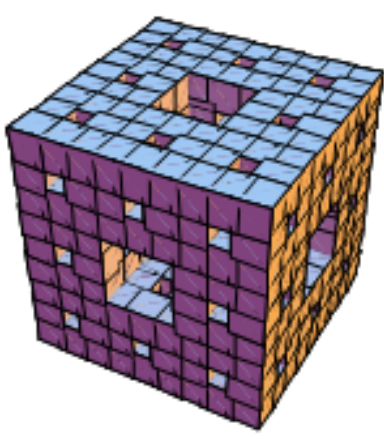

$M_{2\left(2^{\text {nd }} \text { Iteration }\right)}$

Figure 5. Construction of the Menger sponge as IFS

\subsection{Hausdorff Measure and Dimension of the Invariant Set for IFS of the Sierpinski Tetrahedron}

Let $T$ be an invariant set for IFS of the Sierpinski tetrahedron which is defined by the following:

$$
\begin{aligned}
& w_{1}(x, y, z)=\left(\frac{x}{2}, \frac{y}{2}, \frac{(z-\sqrt{3})}{2}\right), \\
& w_{2}(x, y, z)=\left(\frac{x}{2}, \frac{y}{2}+\sqrt{\frac{2}{3}}, \frac{z}{2}-\frac{1}{2 \sqrt{3}}\right), \\
& w_{3}(x, y, z)=\left(\frac{x}{2}-\frac{1}{\sqrt{2}}, \frac{y}{2}-\frac{1}{\sqrt{6}}, \frac{z}{2}-\frac{1}{2 \sqrt{3}}\right), \\
& w_{4}(x, y, z)=\left(\frac{x}{2}+\frac{1}{\sqrt{2}}, \frac{y}{2}-\frac{1}{\sqrt{6}}, \frac{z}{2}-\frac{1}{2 \sqrt{3}}\right)
\end{aligned}
$$

We have 


$$
\begin{aligned}
& \rho\left(w_{1}(x, y, z), w_{1}\left(x^{\prime}, y^{\prime}, z^{\prime}\right)\right) \\
& =\sqrt{\frac{1}{4}\left(x-x^{\prime}\right)^{2}+\frac{1}{4}\left(y-y^{\prime}\right)^{2}+\frac{1}{4}\left(z-z^{\prime}\right)^{2}} \\
& =\frac{1}{2} \sqrt{\left(x-x^{\prime}\right)^{2}+\left(y-y^{\prime}\right)^{2}+\left(z-z^{\prime}\right)^{2}} \\
& =\frac{1}{2} p\left((x, y, x),\left(x^{\prime}, y^{\prime}, z^{\prime}\right)\right)
\end{aligned}
$$

It follows that $w_{1}, w_{2}, w_{3}$ and $w_{4}$ are contraction on

$\mathbf{R}^{3}$ with $L=\frac{1}{2}$.

If $T \subseteq \mathbf{R}^{3}$, then $\bigcap_{i=1}^{4} w_{i}(T)=\phi$ and $L_{i}=\frac{1}{2}$ for each $i=1,2,3,4$. From equation (2), we get

$$
\begin{aligned}
& 4 \cdot\left(\frac{1}{2}\right)^{s}=1 \Rightarrow 2^{s}=4 \Rightarrow s \log 2=\log 4 \\
& \therefore s=\frac{\log 4}{\log 2}=2 .
\end{aligned}
$$

Thus the Hausdorff dimension of the invariant set for IFS of the Sierpinski tetrahedron is $\operatorname{dim}_{H} T=2$.

Since the Sierpinski tetrahedron is in $\mathbf{R}^{3}$, and $\operatorname{dim}_{H} T=2<3$,

$$
\mathcal{H}^{s}(T)=0 \text {. }
$$

Hence the Hausdorff measure of the invariant set for IFS of the Sierpinski tetrahedron is zero.
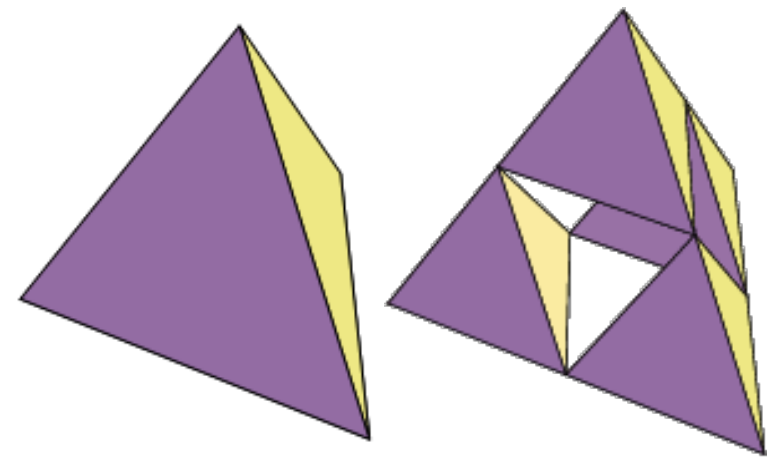

$T_{0}$

$$
\left.T_{1} \text { (1 }{ }^{\text {st }} \text { Iteration }\right)
$$

\section{Conclusions}

We observe that the Hausdorff dimensions of the invariant sets for IFS of the geometric fractals are different. But the Hausdorff measures of the invariant sets for IFS of the geometric fractals are zero as the Hausdorff dimensions are less than the Euclidean dimensions of the corresponding fractals.

\section{REFERENCES}

[1] H. O. Peitger, H. Jungeus and D. Sourpe, Chaos and Fracatls: New frontier of science, Springer, New York, 1992.

[2] M. J. Islam, M. S. Islam, Invariant Measures for Iterated Function Systems of Generalized Cantor Sets, German J. Ad. Math. Sci., Vol. 1(2) (2016), 31-47.

[3] M. F. Barnsley, Fractals Everywhere, Academic Press, Massachusetts, 1993.

[4] J. E. Hutchison, Fractal and self-similarity, Indiana Math. J. 30(1981), 713-743.

[5] R. L. Devaney, a First Course in Chaotic Dynamical Systems, Boston University, Addison-Wesley, West Views Press, 1992.

[6] K. J. Falconer, Fractal Geometry: Mathematical Foundation and Applications, Wiley, New York, 1990.

[7] N. Loughlin, A. Wallis, Fractal Geometry MT4513/5813, 2006. Online available from https://www.students.ncl.ac.u k/n.j.loughlin/fractal2.pdf

[8] P. Lertchoosakul, Introduction of Hausdorff Measure and Dimension, Dynamics Learning Seminar, Liverpool, 2012. Online available from pojlertchoosakul.webs.com/Introduc tionToHausdorffDimension.pdf

[9] M. Hata, On the Structure of Self-similar Sets, Japan J. Appl. Math., 2 (1985), 381-414. 